\title{
Design and Implementation of an Unmanned Tail-sitter
}

\author{
Roman Bapst, Robin Ritz, Lorenz Meier and Marc Pollefeys
}

\begin{abstract}
We present the design and implementation of a small Vertical-Take-Off-and-Landing (VTOL) aircraft. The vehicle requires minimal additional components to achieve the hover capability and is thus very efficient in forward flight. We improve over the state of the art by using a single controller in all flight modes without using blending between hover condition and fixed wing controllers or gain scheduling. We present a compact airflow estimation model for VTOL airframes which rely on the slipstream across control surfaces for hover attitude control. Furthermore we show attitude and position control results in simulation. Finally we show outdoor flight experiments validating our simulation results.
\end{abstract}

\section{INTRODUCTION}

In recent years there has been an increasing amount of applications for Micro Aerial Vehicles (MAV). These applications include aerial photography, precision farming and unmanned cargo. In particular the payload-intense use cases have high requirements on efficiency. The high availability of low-cost inertial sensors and the steady advance in autonomy have made these vehicles attractive for a large audience. A special class of MAVs is characterized by the capability of Vertical Takeoff and Landing (VTOL). The vehicles belonging to this class are able to hover like a helicopter and, therefore, do not require a runway for takeoff or landing. Additionally, they can fly as efficient as a fixed wing aircraft by exploiting the lift generated over a wing surface in forward flight. Hence, these vehicles combine the advantages of both rotary and fixed wing aircraft. The tailsitter rotates its entire body in order to convert from a hover condition into a forward flight condition. This minimizes the mechanical complexity of the actuators in comparison to other VTOL implementations like tilt-wings or tilt-rotors. The latter two implementations rely on rotating either the entire wing or the motors attached to the wing in order to perform a flight transition. However, the high variance of the angle of attack of the wing make this control task more challenging.

The tail-sitter itself can be realized in multiple setups mainly characterized by the method used to create control moments. Oosedo et al. [1] have developed a Quad Rotor Tail-Sitter VTOL UAV without any control surfaces. Therefore, the moments required for attitude control were produced by differential thrust identical to the multicopter case. This method is very effective in hover conditions since strong and rapidly changing moments can be realized. Hough Stone et al. [2] investigated on the T-Wing Tail-sitter UAV which has one engine attached to each wing half. This setup allows to generate a control moment about one axis but in order to control the other two axes the wings are equipped with elevons installed in the slip stream of the propellers.

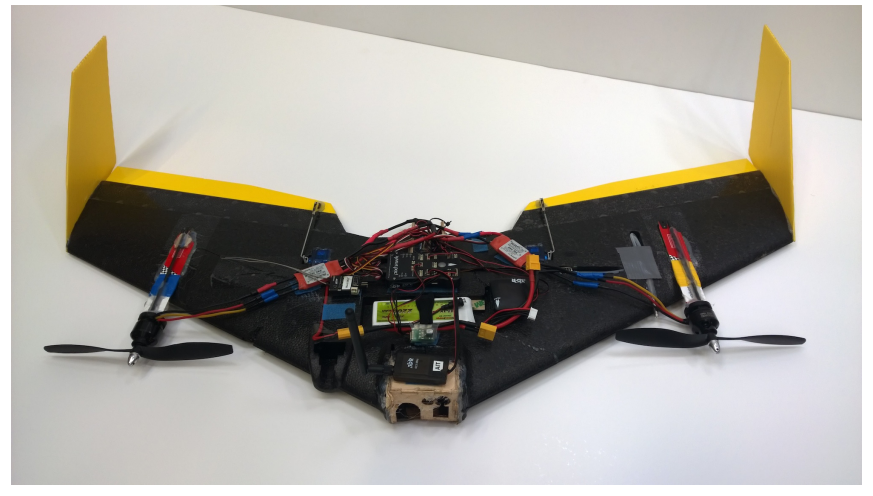

Fig. 1. Tail-sitter VTOL controlled by two propellers and two elevons.

Therefore, attitude control is highly dependent on sufficient airflow stemming from a combination of propeller slipstream and vehicle airspeed. In the past a similar implementation was pursued by Chu et al. [3] who used two engines in a coaxial, counter-rotating configuration with an elevon and a rudder located in the slipstream. All of the above mentioned studies use a similar approach for the control of the vehicle. The flight envelope of the vehicle can be devided into three phases, namely, hover flight, transition and horizontal flight. For all phases conventional controllers such as PID or LQR controllers are utilized to control attitude. The transition phase (front and back transition) is achieved by a transition logic which generates attitude and thrust setpoints for the controller to track. Such a transition logic may embody the steady increase or decrease of the pitch setpoint angle. It becomes clear that the main challenge in the control of all tail-sitter models lies in the drastic, highly nonlinear change in aerodynamic conditions from the hover phase to the horizontal flight phase. Thereby, it is a very challenging task to develop a single controller which is able to stably control the vehicle throughout its entire flight envelope.

In this paper we will discuss the design and implementation of a twin engine tail-sitter model. Focus will be laid on both a simple design and a small size which allows to perform rapid flight tests, increase user safety and minimize flight preparation time. Furthermore, the control architecture for the entire flight envelope of the vehicle will be discussed. A control approach is presented which aims to cover all flight phases of the vehicle with a single controller that uses a physical model of the system in order to cope with the nonlinearities. 


\section{Airframe DESIGN AND MODELling}

\section{A. Airframe Design}

The Caipirinha flying wing from Team Blacksheep was chosen to serve as the base frame for the tail-sitter. The Caipirinha was traditionally designed to fly as a fixed wing plane and uses elevons to control pitch and roll. In order for the Caipirinha model to obtain VTOL capabilities some modifications had to be performed on the original airframe:

- TBS Caipirinha, wingspan $880 \mathrm{~mm}$

- Two brushless motors with 8x45 propellers, CW / CCW

- All-up-weight $0.9 \mathrm{~kg}$, max. $18 \mathrm{~N}$ thrust combined

- Enlarged elevons (300\% of their original surface)

- Pixhawk autopilot (PX4 Stack), airspeed sensor, GPS

- 2200 mAh 60C lithium-polymere battery

The resulting airframe as it is shown in Fig. 1 has both the ability to hover with its nose pointing up and to fly horizontally with its nose pointing forward. In hover flight the airflow produced by the motors mounted on each wing is used to generate a pitching and yawing torque to control attitude. The roll is controlled by applying differential thrust on the motors. In forward flight pitch and roll are controlled by the elevons and yaw is influenced by differential thrust.

\section{B. Vehicle Dynamics}

The dynamic model derived for the simulation and controller design of the Caipirinha tail-sitter is based on firstprinciples and does not reproduce the exact aerodynamic effects acting on the real aircraft. This is mainly due to the complexity of the aerodynamic flows and the lack of an aerodynamic database for this particular model. As a consequence of the simple model, the simulation can serve as a quantitative test bench during the early stages of the controller design, but tuning and small modifications will be needed when performing flight tests on the real airplane. In the following, we introduce the equations of motion for the tail-sitter. If not denoted differently, all vectors are expressed in the body frame of the vehicle.

The conservation of momentum relates the time derivative of the velocity vector $\vec{v}$ to the forces acting on the plane. These forces consist of the gravity force $\vec{f}_{g}$, the lift force $\vec{f}_{l}$, the drag force $\vec{f}_{d}$, the additional lift force stemming from the elevons $\vec{f}_{e}$, and the thrust force $\vec{f}_{t}$. We get

$$
\dot{\vec{v}}=\frac{1}{m}\left(\overrightarrow{f_{g}}+\vec{f}_{l}+\vec{f}_{e}+\vec{f}_{d}+\vec{f}_{t}\right)-\vec{\omega} \times \vec{v},
$$

where $\mathrm{m}$ denotes the mass of the vehicle and $\vec{\omega}$ the angular rate vector. The magnitude of the drag and lift force are computed as

$$
\begin{aligned}
\left\|\overrightarrow{\mathrm{f}}_{\mathrm{l}}\right\|_{2} & =\frac{1}{2} \rho \mathrm{Av}_{\mathrm{air}}^{2} \mathrm{c}_{\mathrm{A}}(\alpha), \\
\left\|\overrightarrow{\mathrm{f}}_{\mathrm{d}}\right\|_{2} & =\frac{1}{2} \rho \mathrm{Av}_{\mathrm{air}}^{2} \mathrm{c}_{\mathrm{D}}(\alpha),
\end{aligned}
$$

where $\rho$ is the density of air, A represents the area of the wing, $\alpha$ the angle of attack, and $v_{\text {air }}$ denotes the reference airspeed $^{1}$. The additional lift created by the elevons at the back side of the wing is modelled similarly to the lift:

$$
\left\|\overrightarrow{\mathrm{f}}_{\mathrm{e}}\right\|_{2}=\frac{1}{2} \rho \mathrm{A}_{\mathrm{e}} \mathrm{v}_{\mathrm{air}}^{2} \mathrm{c}_{\mathrm{E}}(\vec{\delta})
$$

where the elevon lift coefficient $\mathrm{c}_{\mathrm{E}}$ is a function of the elevon command vector $\vec{\delta}$. (Note that $\vec{\delta}$ contains one command for each elevon.) The magnitude of the thrust force is a function of the motor command vector $\vec{\kappa}$ and airspeed $\mathrm{v}_{\text {air }}$ :

$$
\left\|\overrightarrow{\mathrm{f}}_{\text {thrust }}\right\|_{2}=\mathrm{f}\left(\vec{\kappa}, \mathrm{v}_{\text {air }}\right) \text {. }
$$

We now introduce the aerodynamic moment $\overrightarrow{\mathrm{m}}_{\mathrm{a}}$ which is the moment induced by the aerodynamic forces $\vec{f}_{l}, \vec{f}_{d}$ and $\overrightarrow{\mathrm{f}}_{\mathrm{e}}$. Likewise, $\overrightarrow{\mathrm{m}}_{\mathrm{t}}$ denotes the moment induced by the thrust vector $\vec{f}_{t}$. The conservation of rotational momentum is

$$
\dot{\vec{\omega}}=\theta^{-1}\left(\overrightarrow{\mathrm{m}}_{\mathrm{a}}+\overrightarrow{\mathrm{m}}_{\mathrm{t}}-\vec{\omega} \times \theta \vec{\omega}\right)
$$

where $\theta$ denotes the rotational inertia matrix. The time derivative of the quaternion is given by

$$
\dot{\mathrm{q}}=\frac{1}{2} \mathrm{Q} \vec{\omega},
$$

where $\mathrm{Q}$ is the quaternion rate matrix [4]. The time derivative of the position of the plane in the earth frame is

$$
\dot{\overrightarrow{\mathrm{p}}}=\mathrm{R} \overrightarrow{\mathrm{v}}
$$

where $\mathrm{R}$ is rotation matrix from body frame to earth frame and can be computed from the quaternion $\mathrm{q}$.

\section{Airflow Model}

As introduced in Section II-B, both the aerodynamic forces and the propeller thrusts are functions of reference airspeed $v_{\text {air. }}$. In this section we will therefore propose a model for the airflow behind a propeller, based on modelling concepts from [5].

Fig. 2 shows the simplified situation of a propeller moving through the air with airspeed $\mathrm{v}_{0}$ and absorbing the mechanical power $\mathrm{P}$ from the engine it is driven by. The index associated with the symbols of the physical quantities define the location where they hold. The index 1 corresponds to the location just before the propeller disk and the index 2 to the location just after the disk. We assume that the airflow in front and behind the propeller is incompressible and that we can therefore apply Bernoulli's law for incompressible flows. We also model the propeller as a disk of finite diameter and infinitely small thickness. Note that we cannot apply Bernoulli's law across the propeller because there occurs a instantaneous increase of pressure from one side of the propeller to the other. We are looking for an equation which relates the air velocity $\mathrm{v}_{3}$ to the airspeed of the plane $\mathrm{v}_{0}$ and the electrical power consumed by the motor to drive the propeller. Since we are measuring the airspeed of the plane and the power consumption onboard the autopilot we can estimate the total airflow behind the propeller. Considering

\footnotetext{
${ }^{1}$ The reference airspeed $\mathrm{v}_{\text {air }}$ is a combination of the airspeed due to the vehicle's velocity relative to the surrounding air, and the propeller-induced airspeed. In Section II-C, a method to compute $\mathrm{v}_{\text {air }}$ will be presented.
} 


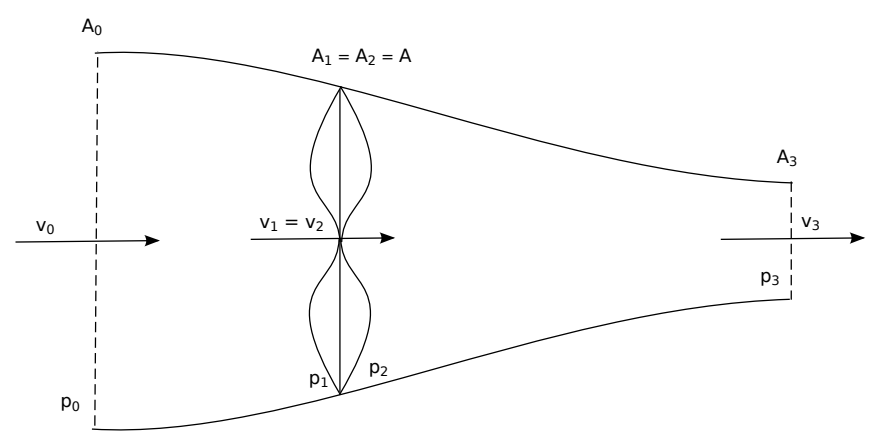

Fig. 2. Modelling airflow as converging tube of accelerated air.

Fig. 2, we apply Bernoulli's law from index 0 to index 1 and from index 2 to index 3 :

$$
\begin{aligned}
& \mathrm{p}_{0}+\frac{1}{2} \rho \mathrm{v}_{0}^{2}=\mathrm{p}_{1}+\frac{1}{2} \rho \mathrm{v}_{1}^{2}, \\
& \mathrm{p}_{3}+\frac{1}{2} \rho \mathrm{v}_{3}^{2}=\mathrm{p}_{2}+\frac{1}{2} \rho \mathrm{v}_{2}^{2} .
\end{aligned}
$$

If we substract (10) from (9) and assume $\mathrm{p}_{3}=\mathrm{p}_{0}$ and $\mathrm{v}_{1}=\mathrm{v}_{2}$, we get

$$
\frac{1}{2} \rho\left(\mathrm{v}_{0}^{2}-\mathrm{v}_{3}^{2}\right)=\mathrm{p}_{1}-\mathrm{p}_{2} .
$$

Assuming a constant density of air we can write the conservation of air mass to be

$$
\mathrm{Av}_{1}=\mathrm{A}_{3} \mathrm{v}_{3} .
$$

The thrust produced by the propeller can be expressed as

$$
\mathrm{T}=\mathrm{A}\left(\mathrm{p}_{2}-\mathrm{p}_{1}\right) .
$$

From this equation we see that the pressure cannot increase continuously across the infinitely thin propeller disk, otherwise the propeller would produce no thrust. If we define a control volume which corresponds to the tube shown in Fig. 2 and apply Newton's momentum theory, we get

$$
\mathrm{T}=\rho \mathrm{A}_{3}\left(\mathrm{v}_{3}-\mathrm{v}_{0}\right) .
$$

Combining (11), (12), (13), and (14), we obtain

$$
\mathrm{v}_{1}=\frac{\mathrm{v}_{0}+\mathrm{v}_{3}}{2} .
$$

We notice that the airspeed at the propeller is the average of the airspeed of the plane and the total airspeed behind the propeller. We introduce the total airspeed increase $\Delta \mathrm{v}$ from point 0 to point 3 :

$$
\Delta \mathrm{v}=\mathrm{v}_{3}-\mathrm{v}_{0} .
$$

Now we can write down the total power absorbed by the propeller $\mathrm{P}_{\text {tot }}$ neglecting all kind of losses:

$$
\mathrm{P}_{\text {tot }}=\mathrm{T}\left(\mathrm{v}_{0}+\frac{\Delta \mathrm{v}}{2}\right) .
$$

The total power is given by the thrust produced by the propeller multiplied by the speed of the air at the propeller.
Next, we define the propeller efficiency $\eta$ as the ratio of propulsive power $\mathrm{P}_{\text {prop }}$ and the total power $\mathrm{P}_{\text {tot }}$ :

$$
\eta=\frac{\mathrm{P}_{\text {prop }}}{\mathrm{P}_{\text {tot }}}=\frac{T v_{0}}{T\left(v_{0}+\frac{\Delta v}{2}\right)}=\frac{v_{0}}{v_{0}+\frac{\Delta v}{2}} .
$$

Using (16) and (18) the total airspeed $\mathrm{v}_{3}$ behind the propeller yields

$$
\mathrm{v}_{3}=\mathrm{v}_{0}+\Delta \mathrm{v}=2 \mathrm{v}_{0}\left(\frac{1}{\eta}-1\right) .
$$

From the last equation it is clear that we can calculate the total airspeed behind the propeller if we know the propeller efficiency and the airspeed of the plane. The efficiency of a propeller is typically given in a diagram as a function of airspeed and various power settings. For the sake of generality, the shape of a typical efficiency function is approximated by the following sigmoid function

$$
\begin{aligned}
& \eta=\frac{2}{1+\mathrm{e}^{\mathrm{av}_{0}}}-1, \\
& \mathrm{a}=\mathrm{b}\left(1-\mathrm{c} \frac{\mathrm{P}_{\mathrm{tot}}}{\mathrm{P}_{\max }}\right) .
\end{aligned}
$$

The parameters $\mathrm{b}$ and $\mathrm{c}$ can be treated as tuning parameters. Note that in practice a minimum airspeed $\mathrm{v}_{0, \min }$ of $1 \frac{\mathrm{m}}{\mathrm{s}}$ was chosen in order to prevent the efficiency to become zero in the denominator of equation 19. Fig. 3 shows the results for the total airspeed estimation behind the propeller for different power and airspeed settings. As we intuitively expect the total airspeed increased with engine power and vehicle airspeed. Notice that for constant motor power the total airspeed eventually increased linearly with airspeed. Also notice that the total airspeed first decreases slightly when the vehicle picks up airspeed from its initial rest position.

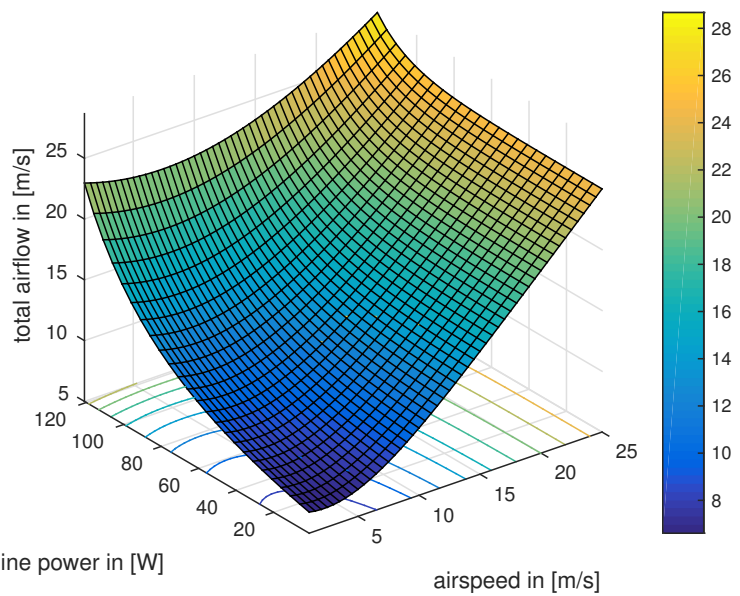

Fig. 3. Two-dimensional map showing resulting airspeed behind propeller as a function of vehicle airspeed and motor power.

\section{CONTROL SYSTEM}

The big challenge in the control of a VTOL UAV lies in the broad flight envelope in which the controller needs to 
control the vehicle. In this section, we introduce a controller which is able to handle the tail-sitter in every flight phase.

There exists plenty of literature dealing with trajectory control of multicopters (see for example [6], [7]). A wellestablished approach is to apply a cascaded control strategy [8]: A desired acceleration is computed based on position and velocity errors of the vehicle. This desired acceleration, together with the desired yaw angle, defines a desired attitude and thrust force. The actuator inputs are then computed by an inner control loop that tracks this desired attitude and thrust setpoint. In this work, we apply a similar control strategy in order to control the tail-sitter throughout its entire flight envelope.

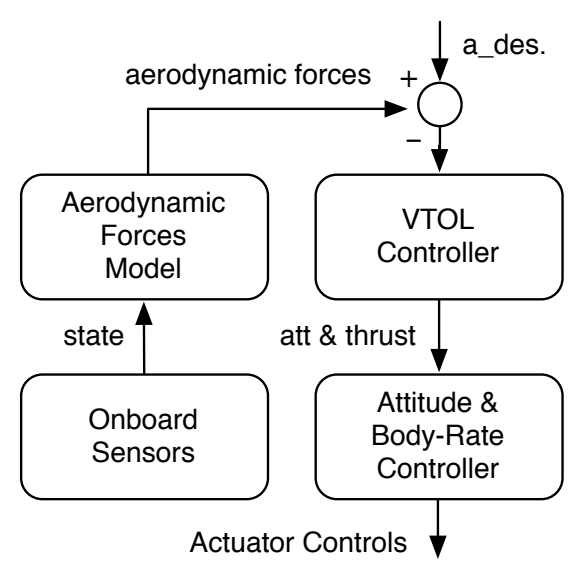

Fig. 4. Block diagram of the VTOL controller. An input reference acceleration is converted to desired attitude and thrust using the aerodynamic model. The outputs are the rotor velocities and elevon deflections.

The main difference between the tail-sitter and a conventional multicopter are the aerodynamic forces in forward flight. The forces acting on a multicopter are dominated by thrust and gravity force, but a tail-sitter is also subject to aerodynamic lift and drag which influences its flight dynamics substantially. While from a controls point of view this appears like a disadvantage, it is exactly what makes the tail-sitter much more efficient than a multicopter in forward flight. We therefore propose a control approach which is based on existing cascaded control schemes for multicopter trajectory tracking, but the controller is extended in order to cope with the additional aerodynamic forces that occur during the various flight phases of a tail-sitter. Fig. 4 depicts the basic structure of the proposed controller.

\section{A. VTOL Controller}

The input to the VTOL controller is the desired proper acceleration $^{2}$. It can be obtained by explicitly demanding a specific acceleration trajectory or by the above mentioned cascaded control loops that convert position and/or velocity errors to a desired acceleration. A model for the aerodynamic properties of the vehicle is applied to compute an estimate of the current aerodynamic forces. The acceleration component caused by these aerodynamic forces is then subtracted

\footnotetext{
${ }^{2}$ In this context, proper acceleration refers to the acceleration relative to free fall, hence it includes the acceleration needed to compensate gravity.
}

from the desired acceleration vector, such that the desired acceleration represents the desired mass-normalized thrust vector. In the following, the vector $\vec{a}_{\text {des }}$ is used to denote the desired acceleration which has already been compensated for the aerodynamic forces.

In order to calculate the desired attitude and thrust, a similar strategy as proposed in [9] is applied. The desired attitude of the vehicle relative to the inertial earth frame is a combination of two rotations:

1) If the desired velocity has a nonzero horizontal component, the yaw angle is chosen such that the nose of the vehicle points towards the desired flight direction. On the other hand, i.e. if the vehicle is hovering or moving along the vertical axis, a desired yaw angle can be chosen freely. This yaw rotation is characterized by the quaternion qyaw.

2) The second rotation has components around the roll and around the pitch axis, and is denoted as qacc. It is computed such that the vehicle's thrust axis is aligned with the desired acceleration vector.

The two rotations can be combined by means of quaternion multiplication [4], in order to obtain the desired attitude of the vehicle:

$$
\mathrm{q}_{\text {des }}=\mathrm{q}_{\mathrm{yaw}} \cdot \mathrm{q}_{\mathrm{acc}} \cdot
$$

The desired thrust force $t_{\text {des }}$ is computed by projecting the desired acceleration vector $\vec{a}_{\text {des }}$ onto the current thrust direction unit vector $\vec{n}_{t}$, i.e.

$$
t_{\text {des }}=\vec{n}_{\mathrm{t}}^{\mathrm{T}} \overrightarrow{\mathrm{a}}_{\mathrm{des}} \mathrm{m} \text {. }
$$

Note that $\mathrm{m}$ denotes the mass of the vehicle. The desired attitude $q_{d e s}$ and thrust setpoint $t_{d e s}$ are then fed to an attitude controller as shown in Fig. 4.

\section{B. Attitude and Body Rate Controller}

The attitude error is given by

$$
\mathrm{q}_{\mathrm{error}}=\mathrm{q}_{\mathrm{est}} \cdot \mathrm{q}_{\mathrm{des}}^{-1},
$$

where qest denotes the current estimated attitude of the vehicle. The desired body rates $\vec{\omega}_{\text {des }}$ are chosen such that the angular error behaves like a first-order system with time constant $\tau_{\mathrm{q}}$ [9]:

$$
\vec{\omega}_{\text {des }}=-\frac{2}{\tau_{\mathrm{q}}} \operatorname{sign}(\text { qerror }, 0) \overrightarrow{\mathrm{q}}_{\text {error }, 1: 3},
$$

where qerror,0 denotes the first element of the error quaternion, and $\overrightarrow{\mathrm{q}}_{\text {error, } 1: 3}$ the vector part, respectively.

We assume time scale separation between attitude and body rate controller, and we design the body rate controller such that the body rates follow three decoupled first-order systems with time constants $\vec{\tau}_{\omega}$. To achieve this, the desired torque acting on the vehicle must be chosen as

$$
\overrightarrow{\mathrm{m}}_{\mathrm{des}}=\theta\left(\vec{\omega}_{\mathrm{des}}-\vec{\omega}_{\mathrm{est}}\right) / \vec{\tau}_{\omega}+\vec{\omega}_{\mathrm{est}} \times \theta \vec{\omega}_{\mathrm{est}},
$$

where $\vec{\omega}_{\text {est }}$ denotes the current measurement of the rate gyro, and the vector division is performed element wise. 
Finally, based on the airspeed model introduced in Section II-B, the actuator inputs (i.e. desired propeller velocities and elevon deflections) are chosen such that the vehicle is subject to the desired torque, i.e. such that $\overrightarrow{\mathrm{m}}_{\mathrm{des}}=\overrightarrow{\mathrm{m}}_{\mathrm{a}}+\overrightarrow{\mathrm{m}}_{\mathrm{t}}$.

\section{RESULTS}

The presented vehicle design was evaluated with a quantitative model in simulation and in real flight experiments. Flight tests were carried out with the Pixhawk ${ }^{3}$ autopilot hardware running the VTOL code as a PX4 Flight Core application [10]. The simulation experiments include a step response which shows that the system is performing the forward and back transition solely based on the the requested trajectory and without any switching logic. The outdoor experiments include forward and back transition in attitude control mode and position control experiments.

\section{A. Simulation}

The dynamic model which was briefly described in Section II was used for a theoretical evaluation of the control method described in Section III. In the simulation, the same aerodynamic model as used by the controller is applied. Hence, the aerodynamic forces are perfectly known to the controller and we thus expect a good tracking behaviour.

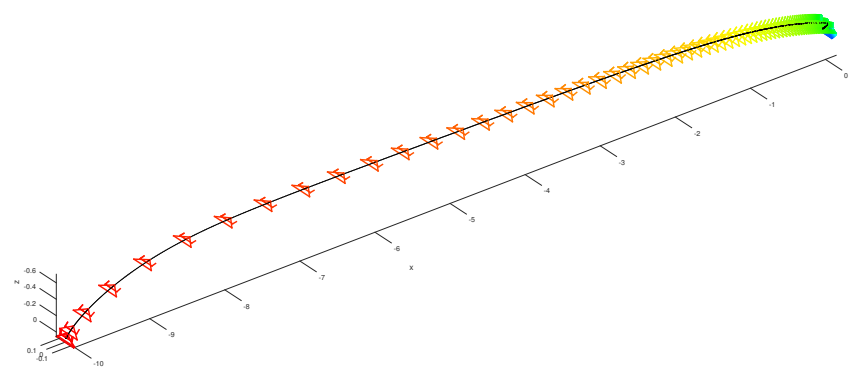

Fig. 5. 3D visualization of the simulation maneuver. The vehicle flies from the left side to the right side in order to reduce the position error. At the beginning and at the end of the maneuver the vehicle is hovering while during the maneuver it flies vertically.

A basic visualization of a simulated maneuver can be seen in Fig. 5. The vehicle is intialized in a hover condition at rest which corresponds to the location at the very left side of Fig. 5. The desired x-position coordinate is then subject to a sudden step such that the new desired position is located at the very right side of Fig. 5. The desired acceleration is computed using proportional gains on position and velocity errors. The vehicle performs a transition from hover to forward flight in order to get to the desired position. As it approaches the target it slowly reduces its forward speed and eventually reaches the target position while hovering.

Fig. 6 depicts the desired and actual position trajectories of the vehicle during the maneuver. Note that the controller is able to reduce the position error in $\mathrm{x}$-direction (red line) without significantly increasing its altitude (orange line) ${ }^{4}$.

\footnotetext{
${ }^{3}$ http://pixhawk.org

${ }^{4}$ Note that we are using a North-East-Down coordinate frame
}

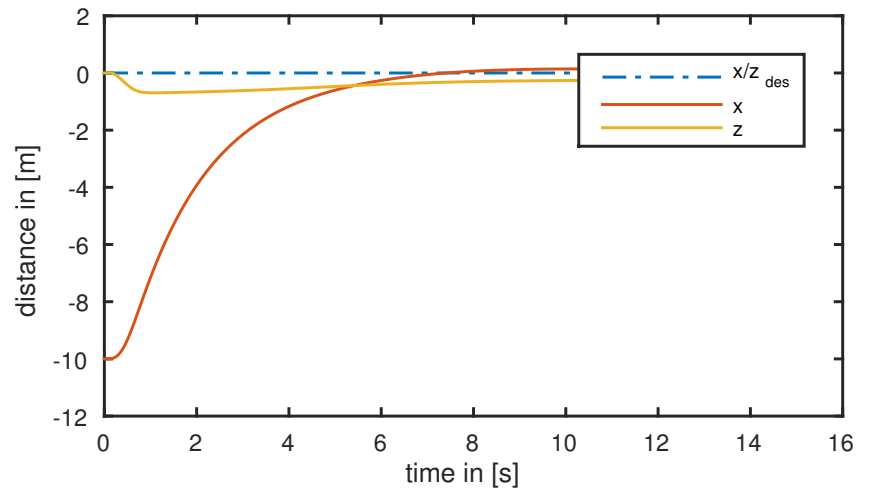

Fig. 6. Desired vs. actual $\mathrm{x}$ - and $\mathrm{z}$-position during the maneuver.

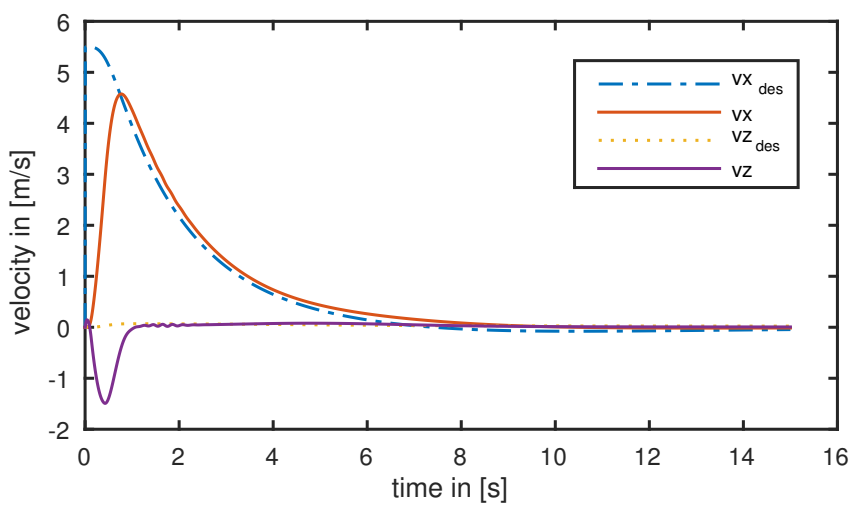

Fig. 7. Desired vs. actual $\mathrm{x}$ - and z-velocity during the maneuver.

The velocity tracking performance can be seen in Fig. 7, and Fig. 8 gives an insight in the pitch tracking performance. All target trajectories are being followed really well.

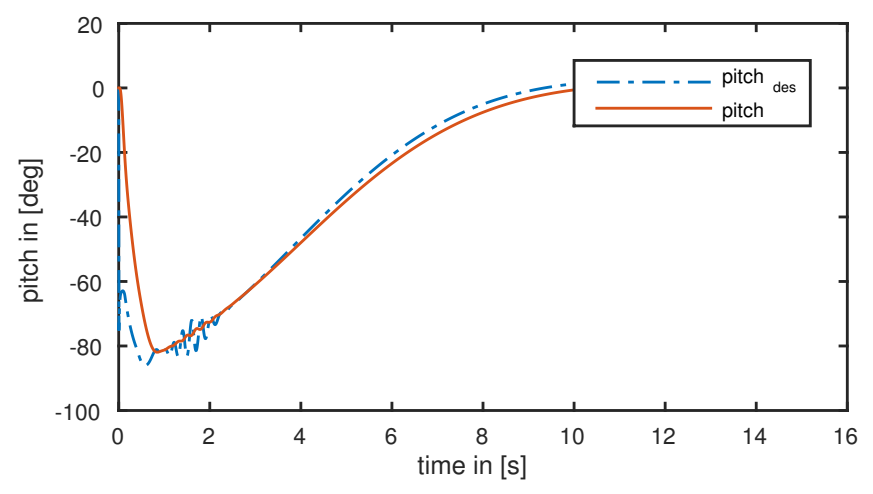

Fig. 8. Desired vs. actual pitch angle during the maneuver.

\section{B. Outdoor Experiments}

The experimental results were obtained in outdoor conditions. All estimation and control was executed on-vehicle with onboard sensing (inertial sensors, digital airspeed sensor and GPS). The flight control code has been published as open source. In the following three types of experiments are presented. In the first experiment the complete flight trajectory was flown, including front- and back transition. The desired velocity trajectory was commanded by the user 
via a remote control but no actual velocity feedback was given to the controller making it open loop. Thus, the controller tried to align it's nose with the desired velocity vector but it did not control the amount of thrust applied. The reason for trying the open loop setup is because the estimated velocity and the measured airspeed are not very accurate at low speeds during the transitions. Fig. 9 shows the pitch control response during the entire flight. The reference is tracked accurately throughout all flight phases, including forward and back transition. From these initial results it is obvious that the performance could be further improved by accurately identifying the airframe and control surface parameters.

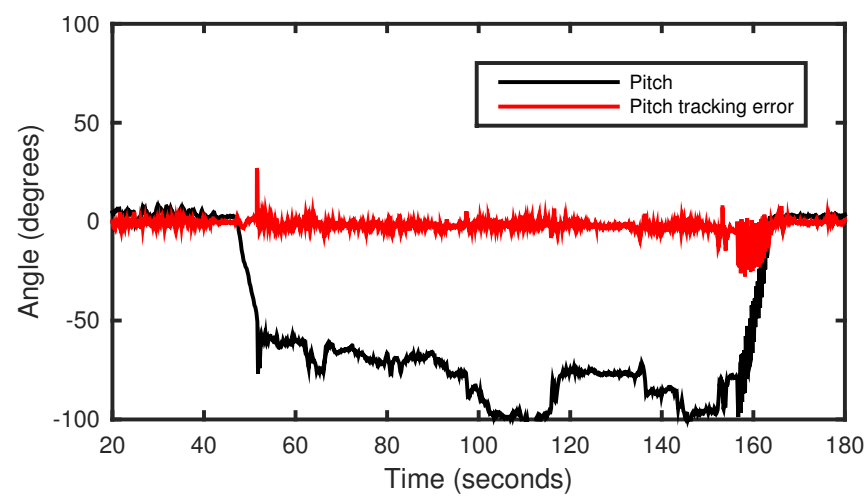

Fig. 9. Pitch angle and tracking error from flight 1 . The plotted angle (black) and error (red / gray) angles show that the controller tracks the reference well throughout the complete flight, including forward and back transition.
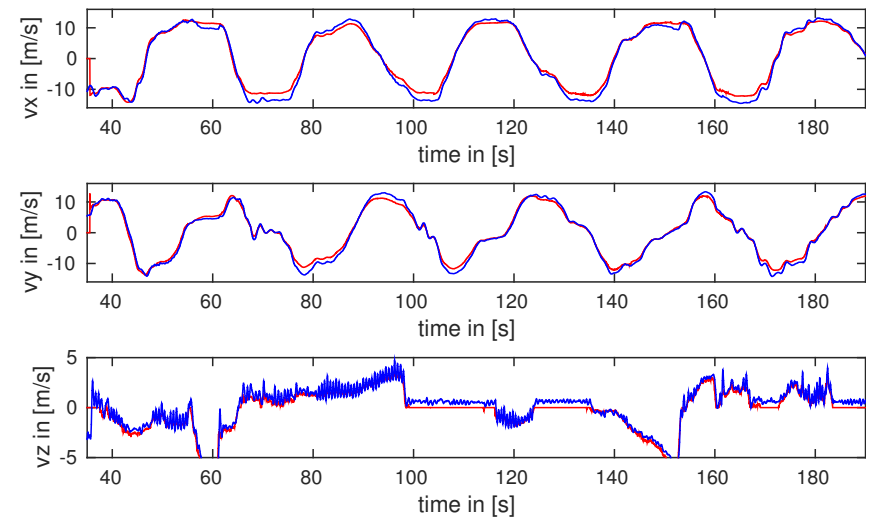

Fig. 10. Closed loop velocity control in three dimensions.

In the second experiment the controller was provided with velocity feedback and therefore, both attitude and thrust were managed by the controller. The user again commanded the desired velocity vector remotely. However, in this experiment the vehicle was just flown in the envelope corresponding to fixed wing flight where the estimated velocity and airspeed are accurate. Figure 10 depicts the velocity tracking performance of the controller. The horizonal velocity components $(\mathrm{x}, \mathrm{y})$ are tracked really well and no oscillations are observable. The vertical velocity component ( $\mathrm{z}$ ) is also tracked well but clearly one can see oscillating behaviour. This can be explained by the high sensitivity of the desired attitude on the current velocity error.

Figure 11 shows the position control performance in hover condition using GPS for closed-loop position control.

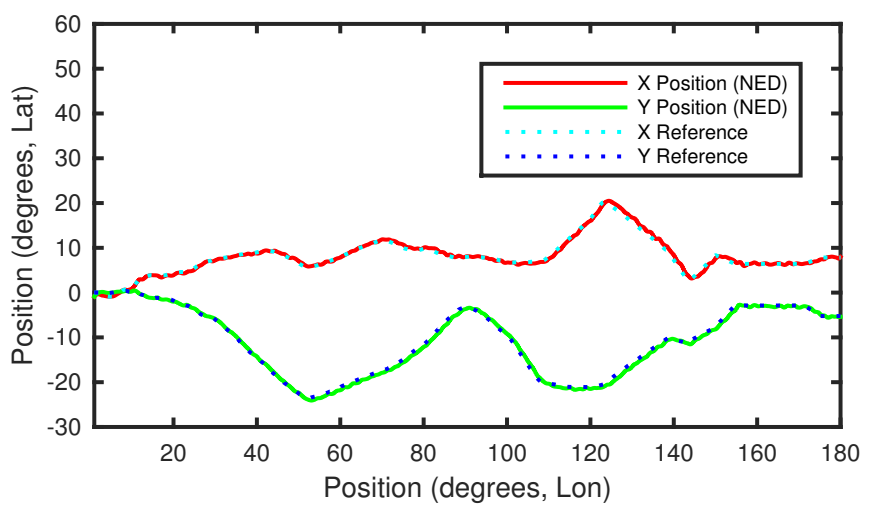

Fig. 11. Position control on flight 2. $\mathrm{X}$ and $\mathrm{Y}$ position control performance in hover condition.

\section{CONCLUSIONS}

We have presented a VTOL controller framework achieving attitude and position control in hover and forward flight using a single controller. The presented total airflow estimation method is an important building block for the further adoption of slip-stream type tail-sitters. The general formulation of the controller enables its use not only for transition control of the VTOL airframe, but general 3D trajectory tracking, which is a promising direction for future work.

\section{REFERENCES}

[1] A. Oosedo, S. Abiko, A. Konno, T. Koizumi, T. Furui, and M. Uchiyama, "Development of a quad rotor tail-sitter vtol uav without control surfaces and experimental verification," in Robotics and Automation (ICRA), 2013 IEEE International Conference on. IEEE, 2013, pp. 317-322.

[2] R. H. Stone, P. Anderson, C. Hutchison, A. Tsai, P. Gibbens, and K. Wong, "Flight testing of the t-wing tail-sitter unmanned air vehicle," Journal of Aircraft, vol. 45, no. 2, pp. 673-685, 2008.

[3] D. Chu, J. Sprinkle, R. Randall, and S. Shkarayev, "Automatic control of vtol micro air vehicle during transition maneuver," in AIAA Guidance, Navigation and Control Conference, 2009, pp. 10-13.

[4] J. Diebel, "Representing attitude: Euler angles, unit quaternions, and rotation vectors," Matrix, 2006.

[5] F. H. Lutze. (2014) Lecture aoe 3104 vehicle performance. Website. [Online]. Available: http://www.dept.aoe.vt.edu/ lutze/ AOE3104/thrustmodels.pdf

[6] D. Mellinger and V. Kumar, "Minimum snap trajectory generation and control for quadrotors," in Robotics and Automation (ICRA), 2011 IEEE International Conference on. IEEE, 2011, pp. 2520-2525.

[7] M. Hehn and R. D'Andrea, "Quadrocopter trajectory generation and control," in IFAC World Congress, 2011.

[8] S. Lupashin, M. Hehn, M. W. Mueller, A. P. Schoellig, M. Sherback, and R. D'Andrea, "A platform for aerial robotics research and demonstration: The flying machine arena," Mechatronics, vol. 24, no. 1, 2014.

[9] D. Brescianini, M. Hehn, and R. D'Andrea, "Nonlinear quadrocopter attitude control," ETH Zurich, Tech. Rep., 2013.

[10] L. Meier, D. Honegger, and M. Pollefeys, "PX4: A node-based multithreaded open source robotics framework for deeply embedded platforms," in Robotics and Automation (ICRA), 2015 IEEE International Conference on, may 2015. 